\title{
Den Business Case für nachhaltige Entwicklung stärken
}

\author{
Wie können geeignete Strategien und Mechanismen zur Förderung von Investi- \\ tionen in integrierten Umweltschutz (cleaner production) in Entwicklungsländern \\ aussehen? Ein Projekt des Umweltprogramms der Vereinten Nationen hat hier \\ in fünf Ländern Erfahrungen gesammelt. Die Resultate geben Hinweise, wie der \\ internationale Finanzsektor durch entsprechende Investitionen in Entwicklungs- \\ ländern einer Sustainable Finance näher kommen könnte.
}

$\mathrm{C}$ Ler Production, im Deutschen oft mit integriertem Umweltschutz übersetzt, wird heute von vielen ExpertInnen als eine wesentliche Voraussetzung für eine nachhaltige Entwicklung gesehen. Lanciert wurde das Konzept 1989 vom Umweltprogramm der Vereinten Nationen (UNEP) als ein neuer und innovativer Ansatz für Ressourcenschonung und Umweltmanagement. Mittlerweile ist Cleaner Production integraler Bestandteil nationaler Umweltpolitiken und betrieblicher Umweltstrategien. Bislang existiert jedoch noch keine klare Definition. Im Wesentlichen beschreibt Cleaner Production eine integrierte und präventive Umweltstrategie, die auf Prozesse, Produkte und Dienstleistungen angewendet wird. Im Gegensatz zu nachgeschalteten End-Of-Pipe-Lösungen führt Cleaner Production zu erhöhter Öko-Effizienz und reduziert Risiken für Mensch und Umwelt. Aus Finanzmarkt- Sicht bietet dieser präventive Ansatz Investoren und Unternehmen daher insbesondere folgende Vorteile:

- Aufbau von Managementkompetenz: Cleaner Production als integraler Bestandteil des betrieblichen Qualitätsmanagements kann sowohl die Produktqualität als auch die Profitabilität des Unternehmen erhöhen.

- Erhöhter Cash Flow und reduziertes Risiko: Cleaner-Production-Strategien, die zum Beispiel die Implementierung eines Umweltmanagementsystems beinhalten, können die Wettbewerbsfähigkeit erhöhen und das Risiko von Umwelthaftungskosten reduzieren.

- Diverse Studien weisen auf den positiven $\mathrm{Zu}$ sammenhang zwischen Umweltleistung eines Unternehmens und dessen finanzieller Performance hin.
Obwohl Investitionen in Cleaner Production finanziell attraktiv sein können, bestehen weiterhin erhebliche Barrieren für diesen Ansatz, nicht nur in den vom Projekt untersuchten Ländern Guatemala, Nicaragua, Tanzania, Vietnam und Zimbabwe. Insgesamt lassen sich sechs Barrieren definieren

\section{- Barrieren in Entwicklungsländern}

- Finanzierung: Es fehlen geeignete Finanzierungsmechanismen, die auf Investitionsprojekte in Cleaner Production zugeschnitten sind, zum Beispiel längere Kreditrïckzahlungsperioden.

- Effizienz: Unter den gegebenen niedrigen Preisen für natürliche Rohstoffe nicht nur in vielen Entwicklungsländern sind Investitionen in Cleaner Production häufig nicht ausreichend kosteneffizient für Unternehmen und Investoren. Ressourceneffizienz wird nicht honoriert.

- Politische Rabmenbedingungen: Die Umweltgesetzgebung ist häufig schwach ausgebildet und bietet Unternehmen wenig Anreize, ihren Fokus auf Ressourcenproduktivität zu lenken.

- Organisation: Umweltmanagement ist in den Unternehmen - wenn überhaupt - schwach aufgestellt.

- Technisch: Zu der Komplexität der Bedarfsermittlung und Implementierung von Cleaner-Production-Maßnahmen kommt hinzu, dass entsprechende Technologien häufig nur sehr eingeschränkt in Entwicklungsländern zur Verfügung steht.

Konzeptionell: Interpretationsprobleme oder Missverständnisse hinsichtlich des Konzepts von Cleaner Production.

Mehrere internationale Organisationen und Entwicklungsbanken haben bereits Projekte zur verstärkten Integration von Cleaner Production in den Industriesektor initiiert und implementiert. Meist geschah dies in Form von technischer Zusammenarbeit und mit Hilfe von Krediten für Investitionen, die von eigens aufgelegten Fonds zur Verfügung gestellt wurden. Diese Projekte waren aber bislang nicht ausreichend, um diese Investitionen aus der Nische in den Mainstream zu holen.

\section{UNEP-Initiativen zur Überwindung der Barrieren}

Im Hinblick auf das Mainstreaming von Nachhaltigkeit im Finanzsektor ist die UNEP Finance Intitiative (1) besonders hervorzuheben, die bereits weltweit über 260 Finanzdienstleister und Versicherer in ein regelmäßiges Forum für nachhaltige Entwicklung zusammenbringt und damit zeigt, dass Investitionen in nachhaltige Entwicklung „good business sense“ sind.

Insbesondere in Entwicklungsländern bestehen jedoch weiterhin Barrieren für Investitionen in nachhaltige Entwicklung. Die Unternehmen investieren vielfach in obsolete Technologien, die Mensch und Umwelt erheblichen Schaden zufïgen und außerdem langfristig hohe Kosten zum Beispiel in Form von Sanierungsmaßnahmen verursachen können.

Um Cleaner Production als eine profitable Investitionsalternative in industriellen Prozessen weiter zu fördern und damit eine Grundlage für eine nachhaltige Entwicklung in Entwicklungsländern zu schaffen, hat UNEP mit finanzieller Unterstïtzung von Norwegen im Jahre 1998 ein Projekt lanciert, das über vier Jahre läuft (2). Die Ziele des Projekts sind:

- Finanzinstitutionen und Unternehmen sollen darin unterstützt werden, die Vorteile von Investitionen in Cleaner Production zu erkennen und die Kreditwürdigkeit von entsprechenden Projekten festzustellen.

- Die Entwicklung und Schaffung neuer und innovativer Finanzierungsinstrumente und -mechanismen zur Förderung von Cleaner Production in Entwicklungsländern.

- Training für Akteure insbesondere aus dem Industrie- und Finanzsektor (Capacity building).

- Das Aufzeigen von Nachfrage nach Finanzierungsinstrumenten für Investitionen in Cleaner Production durch die Erarbeitung eines Portfolios aus profitablen Investitionsprojekten.

- Vorschläge für entsprechende politische Handlungsprogramme, die zum Beispiel über progressive Energiebesteuerung Anreize geben.

Um eine Ausweitung einer solchen Strategie in den Demonstrationsländern und weltweit zu erreichen, versucht das Projekt insbesondere eine 
effektivere Interaktion von Finanzinstitutionen und Unternehmen und anderen Stakeholdern wie den Medien herbeizuführen. Dazu hat das UNEPProjekt in Zusammenarbeit mit einem internationalen ExpertInnen-Netzwerk ein Set von Trainingskursen erarbeitet, die derzeit zunächst in den fünf Demonstrationsländern implementiert werden und dann international Verwendung finden sollen. Sie richten sich zum einen insbesondere an Finanzanalysten und Finanzdienstleister. Zum anderen werden Kurse für Unternehmen und Industrievertreter angeboten, die stärker auf die Entwicklung von betrieblichen Investitionsprojekten im Bereich Cleaner Production eingehen, die aus Sicht von Finanzdienstleistern attraktive Investments darstellen könnten.

\section{Mainstreaming Cleaner Production}

Durch die den eigentlichen Trainingskursen vorgeschalteten interaktiven finanzwirtschaftlichen Trainingseinheiten in den Demonstrationsländern gelang der Aufbau einer nationalen ExpertInnengruppe, die bereits erfolgreich Investitionsprojekte entwickelt hat. Diese Projekte münden in ein Portfolio kreditwürdiger Anträge und signalisieren so den Finanzdienstleistern, dass Nachfrage nach Finanzierungsinstrumenten für CleanerProduction-Projekte besteht. Zudem bieten die Investitionsprojekte Anlegern profitable langfristige Ertragschancen im wachsenden Sektor des Sustainable Investment.

Damit ist bereits ein Dialog von Unternehmen und Finanzdienstleistern in den fünf Ländern initiiert. Für das nun angelaufene Trainingsprogramm liefern sie reale Fallstudien. Bedarfserhebungen in den fünf Ländern ermöglichten zusätzlich eine grö- ßere Tiefenschärfe und Zielgruppenorientierung der Kurskonzepte (3). Mit der Veröffentlichung der Ergebnisse auf internationaler Ebene soll in $\mathrm{Zu}$ kunft außerdem eine noch stärkere Verknüpfung mit der UNEP Finance Initiative, etwa im Rahmen eines Sustainable-Financing-Netzwerk, stattfinden. Projekte wie das beschriebene UNEP-Projekt schaffen ein starkes Signal für Akteure aus dem Industrie und Finanzsektor, dass Investitionen in Cleaner Production auch in Entwicklungsländern profitabel sind. Dies fördert die Evolution des Konzepts der Nachhaltigkeit in den internationalen Finanzmärkten. Gleichzeitig bedeutet eine Etablierung von präventiven Strategien im Industriesektor der aufstrebenden Märkte Schritte in eine nachhaltigere Zukunft.

\section{Anmerkungen}

(1) Mehr Informationen zu der UNEP Finance Initiative im Internet unter www.unep.ch/etu/fi/index.htm

(2) Mehr Informationen unter der Projektwebsite http:// www.financing $\mathrm{CP}$.org

(3) Die vorbereitende Studie kann per E-mail über ari.huhtala@unep.fr angefordert werden. Teile der Studie befinden sich auch auf der Projektwebsite. Der Inhalt der laufenden Kurse werden Ende 2001 in Form eines Handbuches frei zugänglich gemacht.

\section{Der Autor}

Niki Rosinski arbeitet als Sustainability Analyst bei Sustainable Asset Management (SAM) in Zürich und ist Mitglied des Sustainable Europe Research Institute (SERI).

Kontakt: SAM, Zollikerstrasse 60, CH-8702 Zollikon-Zürich, Tel. 0041-1-39710-10, Fax -80, E-mail: niki@sam-group.com

\section{Neu im ökom verlag Gute Ideen,} aber niemand kennt sie?

Veröffentlichen Sie deshalb Ihre zündenden Ideen, Ihre wegweisenden Forschungsergebnisse, neuen Anregungen im ökom Verlag, der Plattform für Ökologie und Nachhaltigkeit!

Mit seinem neuen Buch-Programm bietet Ihnen der ökom Verlag jetzt die Möglichkeit, Ihre Publikation zum Thema Nachhaltigkeit, Ökologie und Umweltschutz einem breiten und interessierten Publikum vorzustellen. Denkbar sind Dissertationen, Doktor- und Forschungsarbeiten, Habilitationen, Tagungsberichte oder Seminarunterlagen.

$$
\text { Ihre Gedanken sind uns die Mühe wert! }
$$

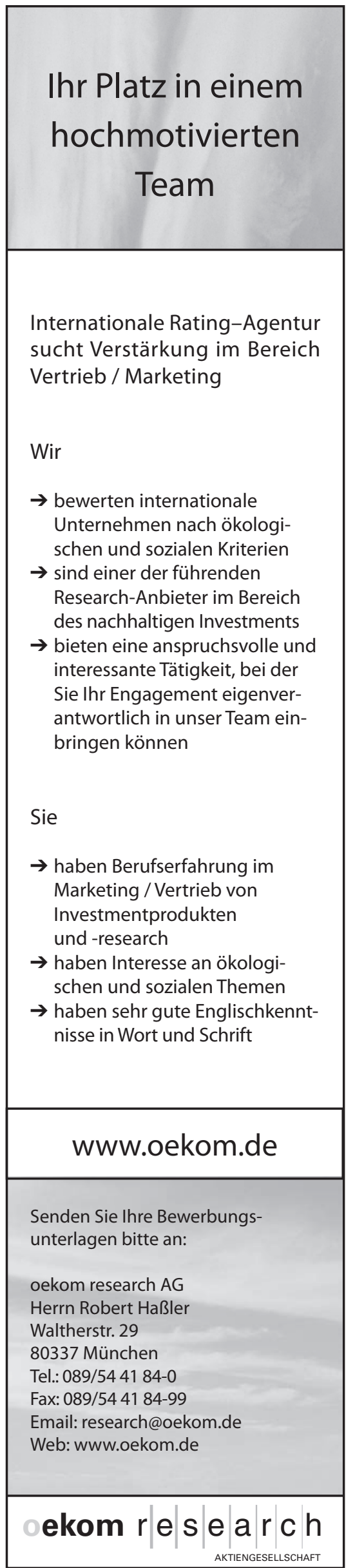


(c) 20I0 Authors; licensee IÖW and oekom verlag. This is an article distributed under the terms of the Creative Commons Attribution Non-Commercial No Derivates License (http://creativecommons.org/licenses/by-nc-nd/3.o/), which permits unrestricted use, distribution, and reproduction in any medium, provided the original work is properly cited. 